\title{
Simulation of the Building Materials Production Process
}

\author{
Olga Vasil'eva* \\ Moscow State University of Civil Engineering, Yaroslavskoe shosse, 26, Moscow, 129337, Russia
}

\begin{abstract}
We study characteristics and properties of modified GodunovSultangazin kinetic system solution allows us to describe the nature and time of technological processes for the production of building materials based on autocatalysis reaction. This system is nonlinear, which explains the difficulty of its analytical solution. So, we use the numerical method of its investigation. The numerical results obtained and analyzed.
\end{abstract}

\section{Introduction}

Discrete kinetic systems, such as the Carleman system, the Godunov-Sultangazin system, the Broadwell system, are used to simulate reversible chemical reactions of autocatalysis with a different number of reagents [1,2]. The study of characteristics and properties of solutions systems allows us to describe the nature and time of technological processes for the production of building materials based on autocatalysis reactions. We consider the evolution and characteristics of the harmonic perturbation of the equilibrium position, described by the initial-boundary value problem for the Godunov-Sultangazin system. The Godunov-Sultangazin system describes the interaction of three different components.

This system is nonlinear, which explains the difficulty of its analytical solution. Therefore, we use numerical methods for its investigation. For convenience of analysis, the system is formulated for the reduced variables. For the numerical solution of the problem, we apply a finite-difference method of the second-order accuracy. To study the parameters and characteristics of the numerical solution we use linear interpolation and quadrature formulas of the second order of accuracy. The results of the numerical study are given.

\section{Formulation of the problem}

We consider the initial-boundary problem for modified Godunov-Sultangazin system [1]

$$
\begin{aligned}
& u_{t}+c u_{x}=\varepsilon^{-1}\left(v^{2}-u w\right), \quad t>0,0<x<1 \\
& v_{t}=-2 \varepsilon^{-1}\left(v^{2}-u w\right), \\
& w_{t}-c w_{x}=\varepsilon^{-1}\left(v^{2}-u w\right),
\end{aligned}
$$

with initial-boundary conditions of a special type

\footnotetext{
* Corresponding author: vasilievaoa@mgsu.ru
} 


$$
\begin{gathered}
u(0, x)=1+A \sin 2 \pi \omega x, \\
v(0, x)=1+A \sin 2 \pi \omega x, \\
w(0, x)=1-A \sin 2 \pi \omega x, \\
u(t, 0)=1, w(t, 1)=1 .
\end{gathered}
$$

Here $u(t, x), v(t, x)$, and $w(t, x)$ are the densities of the particles of the reagents of three types that are interacting in a narrow vessel of length 1 . Particles of the first and third types move with constant oppositely directed velocities equal in modulus $c$. Particles of the second type move at zero speed. The stationary solution of the problem is the position of the equilibrium, in which the reaction stops, stabilization occurs. On the left boundary, the density of particles of the first type is equal to 1 . On the right boundary, the density of the third-type particles is equal to 1 .

We investigate the character and time of stabilization process. To estimate the degree of stabilization we introduce the following deviation functions:

$$
\begin{aligned}
E_{u}(t) & =\left(\int_{0}^{1}\left(u(t, x)-u^{s}(x)\right)^{2} d x\right)^{\frac{1}{2}}, \\
E_{v}(t) & =\left(\int_{0}^{1}\left(v(t, x)-v^{s}(x)\right)^{2} d x\right)^{\frac{1}{2}}, \\
E_{w}(t) & =\left(\int_{0}^{1}\left(w(t, x)-w^{s}(x)\right)^{2} d x\right)^{\frac{1}{2}}, \\
E(t) & =\left(E_{u}{ }^{2}(t)+E_{v}{ }^{2}(t)+E_{w}{ }^{2}(t)\right)^{0.5} .
\end{aligned}
$$

The maximum deviations of the solution components from stationary solution $u^{s}(x)=1$, $v^{s}(x)=1, w^{s}(x)=1$ denote by

$$
\begin{gathered}
D u(t)=\max _{0 \leq x \leq 1}|u(t, x)-1|, \\
D v(t)=\max _{0 \leq x \leq 1}|v(t, x)-1|, \\
D w(t)=\max _{0 \leq x \leq 1}|w(t, x)-1| .
\end{gathered}
$$

\section{Numerical results}

We consider the problem (1) - (2) for the $c=0.05, \varepsilon=0.005, A=0.6, \omega=10$. Figure 1 presents initial condition of problem $u(0, x)=v(0, x), w(0, x)$. Figures $2-5$, show numerical solution of problem $u\left(t_{i}, x\right), v\left(t_{i}, x\right)$ and $w\left(t_{i}, x\right)$, the values of the time variable $t_{1}=5, t_{2}=10$, $t_{3}=15, t_{4}=20$. Figure 6 demostrates the maximum solution deviations from equilibrium state $D u(t), D v(t), D w(t)$. The dependences have the oscillating character.

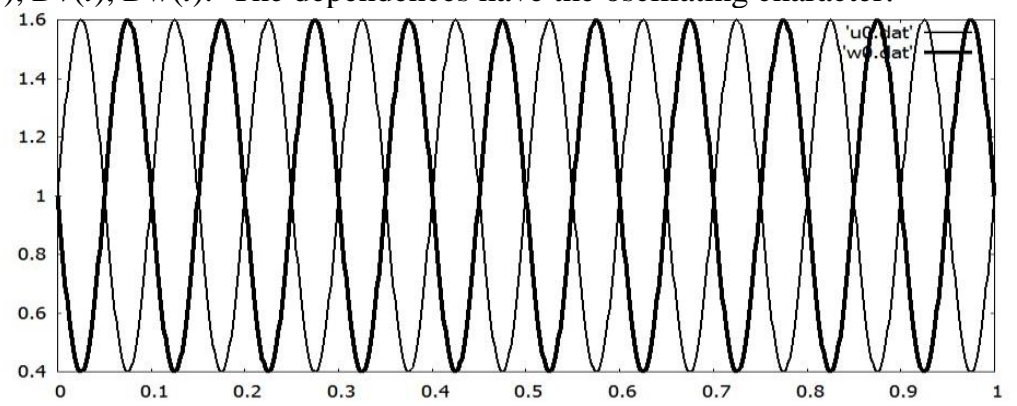

Fig. 1. The initial conditions $u(0, x)=v(0, x)$ and $w(0, x)$ 


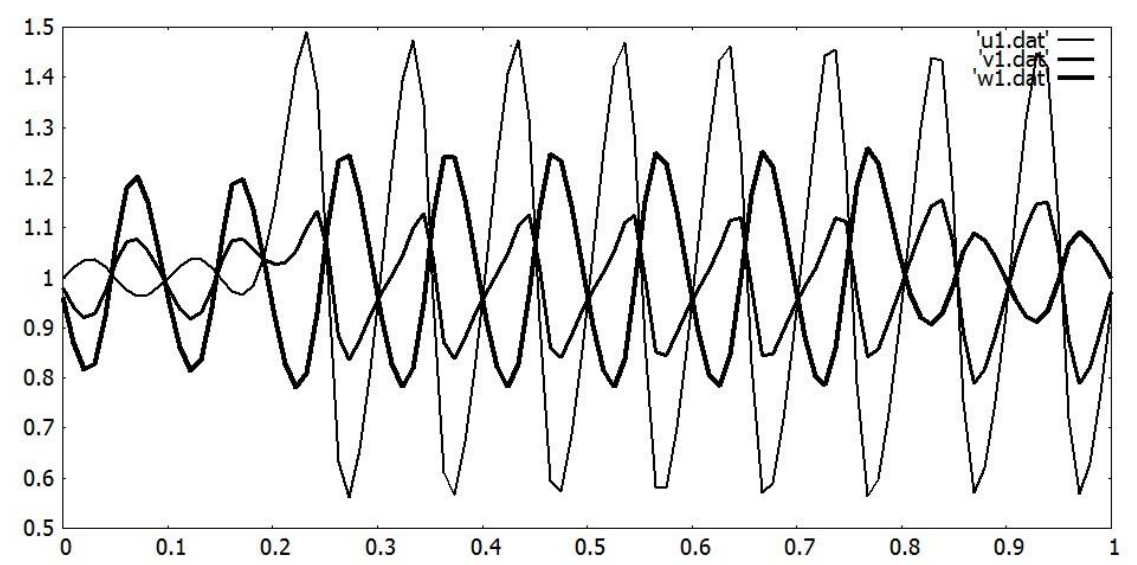

Fig. 2. The solution $u(5, x), v(5, x)$ and $w(5, x)$

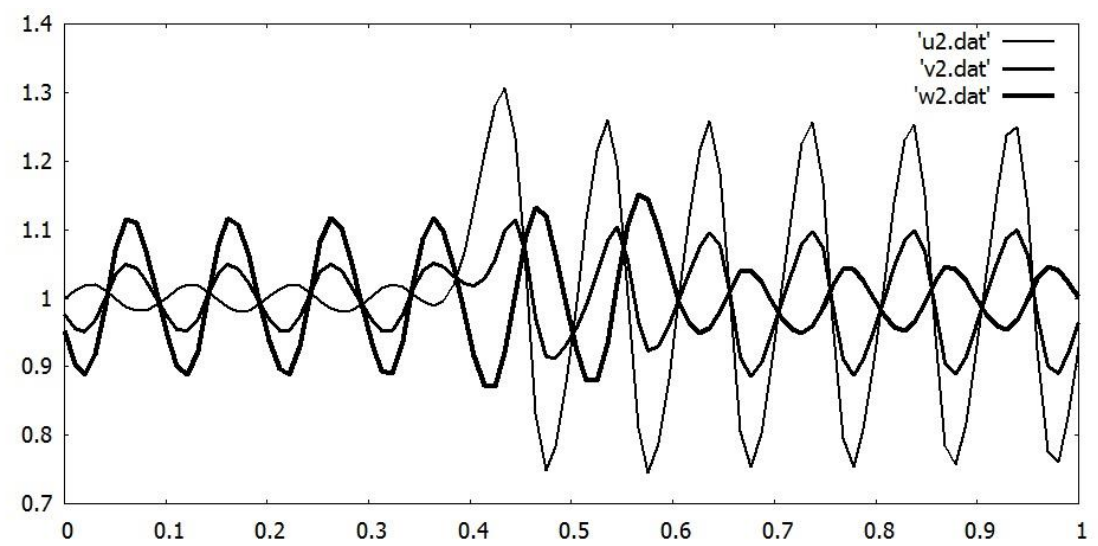

Fig. 3. The solution $u(10, x), v(10, x)$ and $w(10, x)$

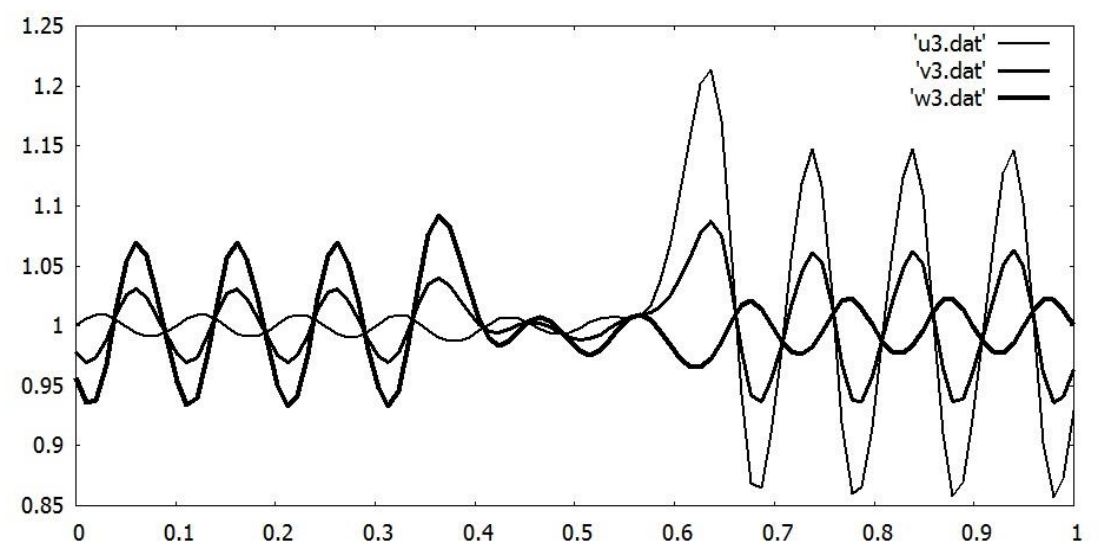

Fig. 4. The solution $u(15, x), v(15, x)$ and $w(15, x)$ 


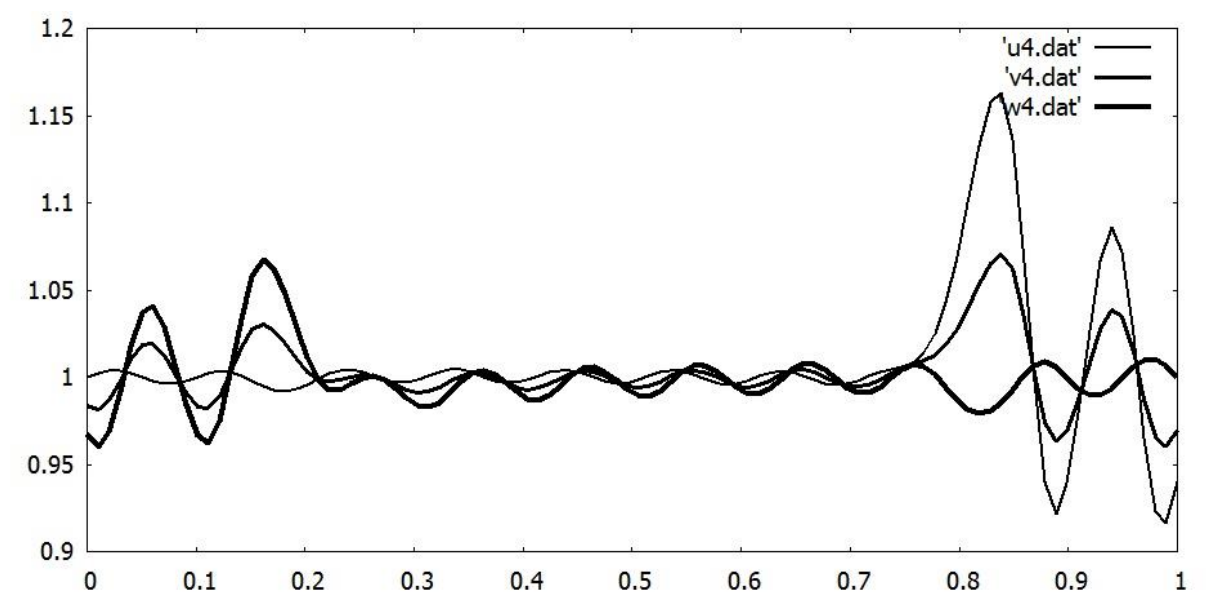

Fig. 5. The solution $u(20, x), v(20, x)$ and $w(20, x)$

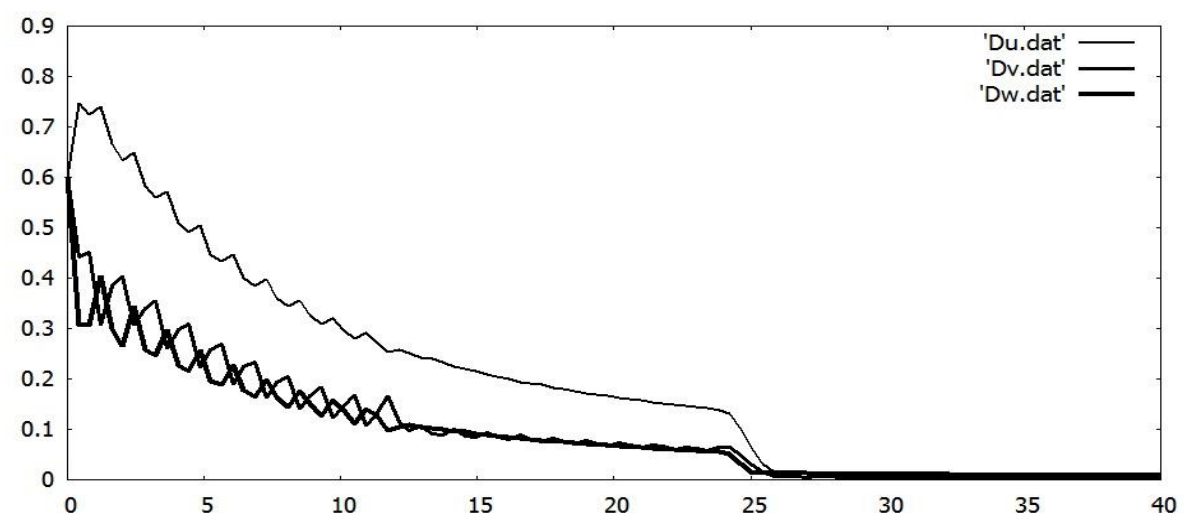

Fig,.6. The maximum solution deviations from equilibrium state $D u(t), D v(t), D w(t)$

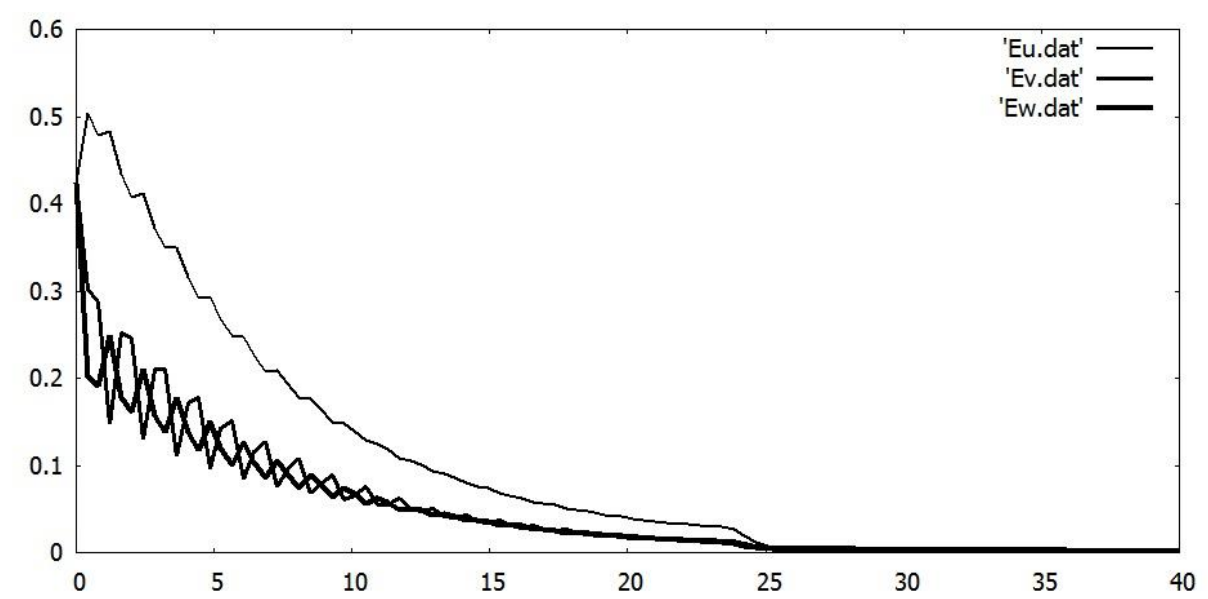

Fig. 7. The deviations $E_{u}(t), E_{v}(t)$ and $E_{w}(t)$ 


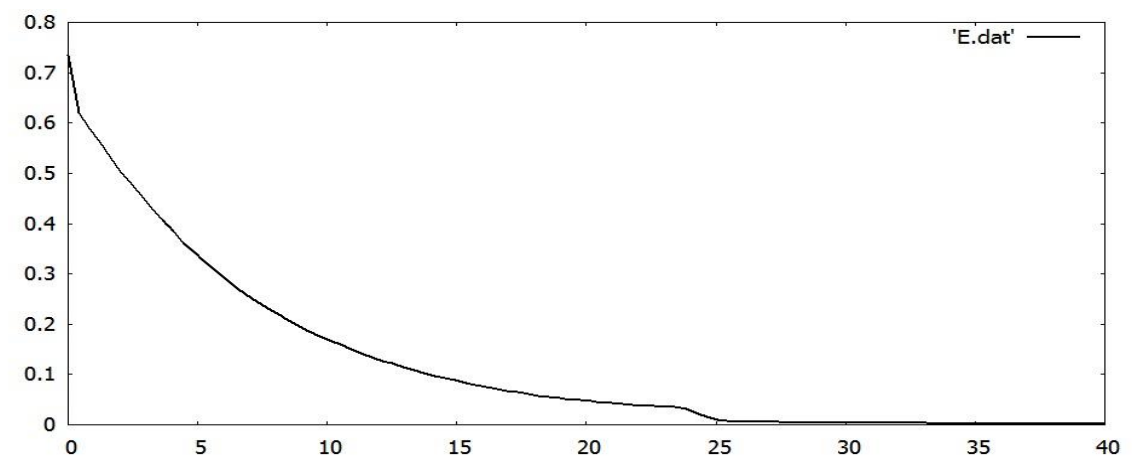

Fig. 8. The total deviation $E(t)$

Figure 7 demonstrates the deviations of the solution components. The tendency to zero of the deviations of each of the components of the solution has a nonmonotonic oscillatory character, which demonstrates multiple mutual transitions of particles from one type of reagent to another (reversibility of the reaction). Figure 8 represents the total deviation of the solution from the equilibrium position $E(t)$. The total deviation tends monotonically to zero and at $t=25$ practically equal zero (completion of the reaction).

Figures 9-11 show the phase planes for different pairs of solution components. In all three cases, the tendency to $(0,0)$ has an oscillatory character with different decreasing amplitudes.

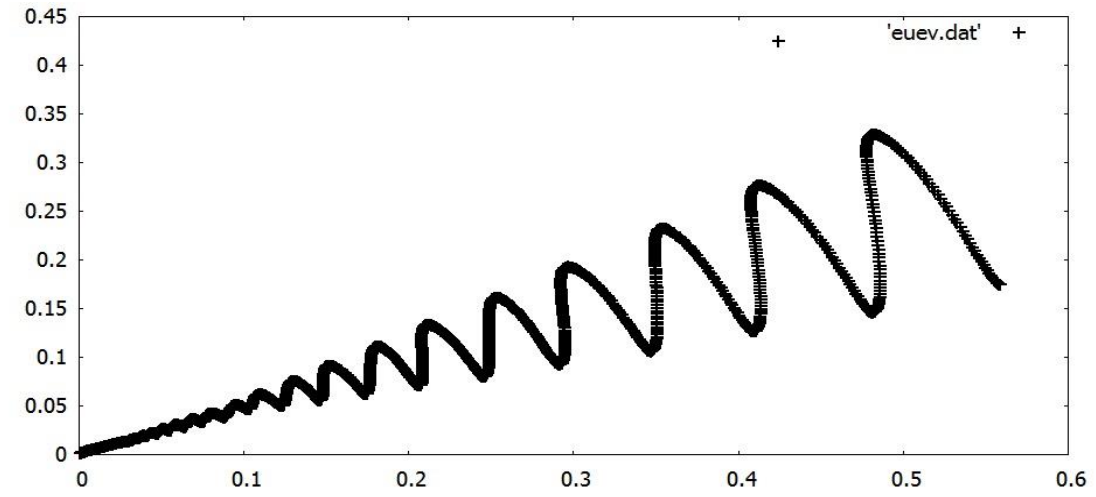

Fig. 9. The phase plane $\left(E_{u}(t), E_{v}(t)\right)$

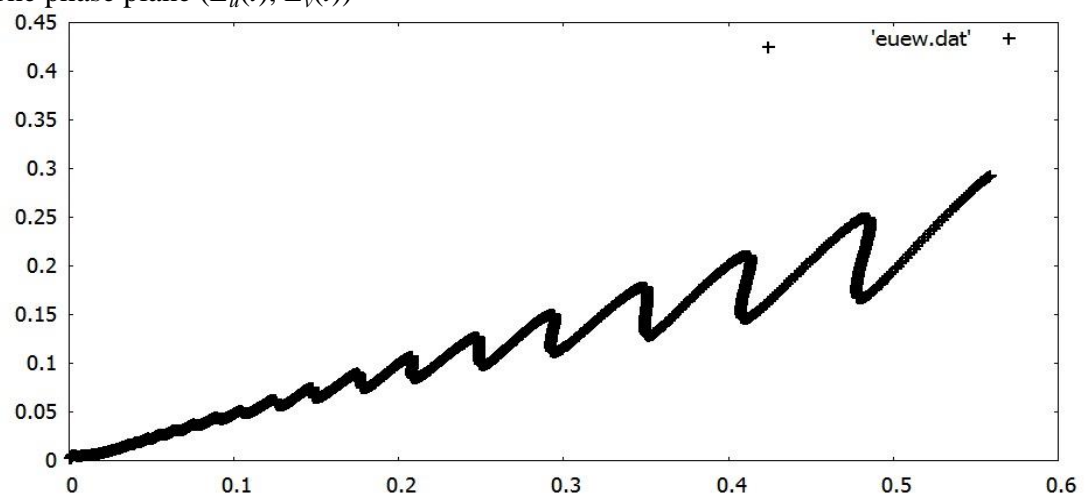

Fig. 10. The phase plane $\left(E_{u}(t), E_{w}(t)\right)$ 


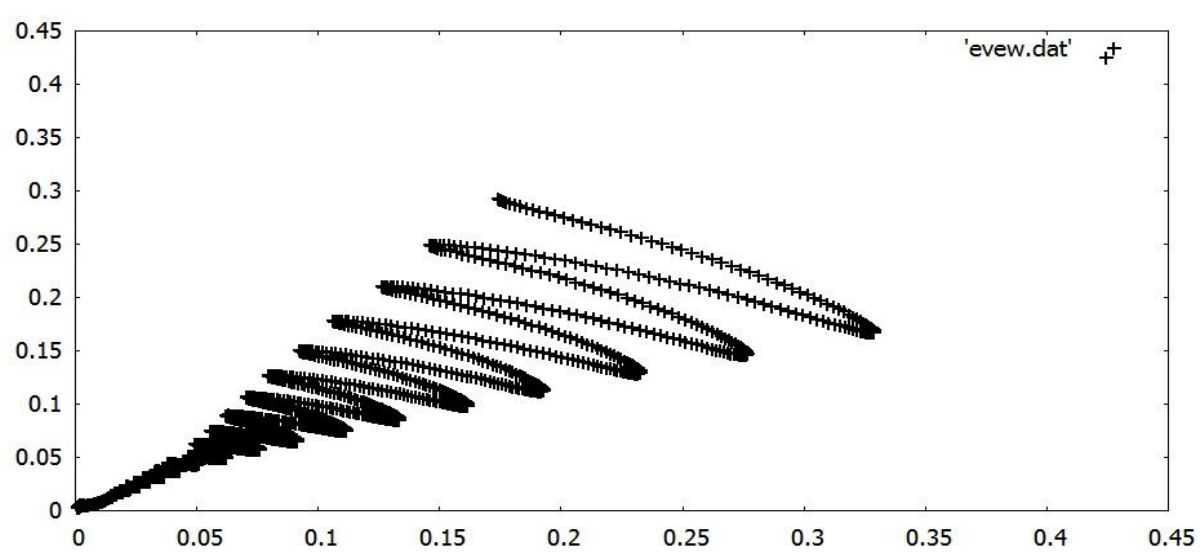

Fig. 11. The phase plane $\left(E_{v}(t), E_{w}(t)\right)$

\section{Conclusion}

We have considered the kinetic system of equations for the mathematical description of the reversible reaction of autocatalysis. The evolution of the harmonic perturbation of the equilibrium state is numerically investigated. Stabilization of the process and its character are investigated. An estimate of the reaction time is obtained. The long reaction time can be explained by the small speed of the reagent particles, as well as by the special choice of the initial distribution of reagent densities in the vessel. Of particular interest from a practical point of view is the random distribution of initial reagent densities, which is the subject of the next article.

The author would like to express gratitude to Professor E.V.Radkevich for formulation of the problem and useful discussions and comments.

\section{References}

1. S. K. Godunov, U. M. Sultangazin. Math. Surv. 26, 3 (1971)

2. V. V. Aristov, O.V. Ilyin. AIP Conference proceeding. $27^{\text {th }}$ International Symposium on Rarefied Gas Dynamics - 2010, RGD27. 649-654 (2011)

3. E. V. Radkevich, O.A.Vasil'eva, J. Math. Sci. 224, 5 (2017)

4. E. V. Radkevich. J. Math. Sci. 181, 5 (2012)

5. E. V. Radkevich. J. Math. Sci. 184, 4 (2012)

6. O.A.Vasil'eva. Vestnik MGSU. 4, 27 (2016)

7. O. Vasil'eva. Proc. Engineering. 153, 825 (2016) 